\title{
Thermo-mechanical analysis of retro-reflectors for interferometry and polarimetry in $\mathrm{W} 7-\mathrm{X}$
}

\author{
M. Köppen ${ }^{\mathrm{a}}$, M. Hirsch ${ }^{\mathrm{a}}$, J. Ernst ${ }^{\mathrm{a}}$, W. A. Vliegenthart ${ }^{\mathrm{b}}$, V. Bykov ${ }^{\mathrm{a}}$, F. Schauer ${ }^{\mathrm{a}}$, M. Y. Ye ${ }^{\mathrm{a}}$ \\ ${ }^{a}$ Max-Planck-Institut für Plasmaphysik, Euratom Association, Teilinstitut Greifswald, \\ Wendelsteinstr. 1, 17491 Greifswald, Germany \\ ${ }^{b}$ TNO Science \& Industry, NL-2600 AD, Delft, Netherlands
}

\begin{abstract}
The stellarator Wendelstein 7-X (W7-X) is presently under construction at the Max-Planck-Institut für Plasmaphysik in Greifswald, Germany. The plasma density profile will be measured by two-colour interferometry where for each line of sight through the plasma the phase shifts of two far-infrared laser beams are compared. Due to the 3D shape of the superconducting coils in W7-X, opposite ports are not available. Instead, corner cube retroreflectors (CCR) will be incorporated in the plasma vessel heat shield that reflect the beams back to the entrance window and diagnostic. This requires high accuracy of the reflecting surfaces which will be subjected to thermal loads from plasma radiation and therefore be heated up, displaced and deformed during operation.

ANSYS is used to perform coupled thermo-mechanical analyses of the CCR, including plasma radiation, thermal conduction, and radiation between all parts. The resulting displacements provide the inputs to MATLAB routines which then yield local deformations, curvature radii, and tilting of the reflecting surfaces. This information is used to optimize the design of the CCR and their fixations.
\end{abstract}

Keywords: W7-X, retro-reflectors, thermo-mechanical, FE-analysis, interferometry, polarimetry

\section{Introduction}

The plasma density profile of the stellarator Wendelstein 7-X (W7-X) will be measured via the phase shift of two far-infrared-laser beams (lambda $=10.6 \mu \mathrm{m}$ and $5 \mu \mathrm{m}$ ) which pass through the plasma. Due to the shape of the modular coils and the divertor it is geometrically not possible to launch and detect the laser beams at opposite positions of the machine. Instead, corner cube retro-reflectors (CCR) will be used which have to be incorporated in the plasma vessel heat shield and will reflect the beams back to the diagnostic [1]. Preliminary investigations for an initial design of the retro reflectors were already reported in [2,3]. The improved design avoids mutual stresses in the reflector plates under heat load. The CCR consists basically of three elements, namely a cube body and two plates. Due to the thermal loads from plasma radiation it will be displaced and deformed during operation. While moving the whole CCR has little influence on its functionality, it is important to keep local deformations of the reflecting plates and angle deviations between the three surfaces to a minimum in order to achieve the required optical accuracy.

This paper investigates how CCR surfaces used in W7-X deform and tilt due to thermal loads. In section 2 it is shown in general how different cooling arrangements can lead to different curvatures in the reflecting surfaces of CCRs. Results of coupled thermomechanical simulations for the W7-X - CCRs are presented in section 3. This also includes an overview of resulting curvatures and tilting angles of the CCR plates. Section 4 briefly shows how changes in the shape of thermal contacts to the heat sink can be used to maximize the curvature radii of the CCR surfaces and thus to improve the design.

\section{Basic investigations}

The major disturbance to the CCR's function will be due to curvatures of the reflecting surfaces. A small gap avoids mutual stresses between plates which otherwise would become the main cause for thermally induced deformations [3]. The main influence will therefore come from temperature gradients across each single plate that lead to different thermal expansions at the surfaces. With constant plasma radiation, it is still possible to get different temperature distributions in the plates, depending on the type of cooling employed. This section investigates two different kinds of cooling leading to different maximum curvature radii and therefore different spurious deviations of the reflected beams.

\subsection{One dimensional heat transfer}

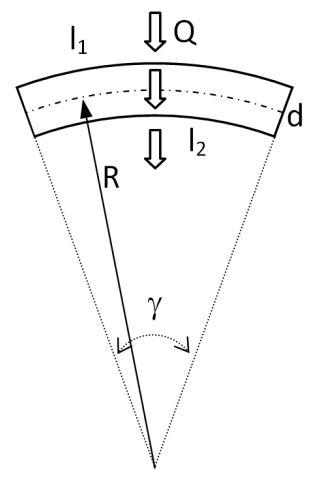

Fig. 1 Curvature due to 1D heat transfer 
A temperature difference develops across the thickness of a plate which is heated from the top, e.g. due to plasma radiation, and cooled from below either due to radiation or conduction. This temperature difference determines the curvature of the plate due to different thermal expansions of the top and bottom surfaces (see Fig. 1). Neglecting thermal stresses, the curvature along a symmetry line of this plate is given by the following equations.

$$
\begin{aligned}
& \gamma=\frac{l \cdot \alpha \cdot\left(T_{1}-T_{2}\right)}{d} \\
& \Delta T=q \frac{d}{\lambda} \\
& \gamma=\frac{l \cdot \alpha \cdot q \cdot d}{d \cdot \lambda} \\
& R=\frac{l}{\gamma} \\
& 1-\text { length }=\left(1_{1}+1_{2}\right) / 2 \\
& \alpha-\text { coefficient of thermal expansion } \\
& \mathrm{T}-\text { Temperature } \\
& \mathrm{d}-\text { thickness of the plate } \\
& \mathrm{q}-\text { heat flux } \\
& \lambda-\text { thermal conductivity } \\
& \gamma-\text { angle of curvature } \\
& \mathrm{R}-\text { radius of curvature }
\end{aligned}
$$

While equation 1 shows that the curvature $1 / R=\gamma / 1$ always depends on the temperature difference across the plate, equation 3 is illustrating that in the special case of $1 \mathrm{D}$ heat transfer the curvature is only a function of the heat flux and the ratio of thermal expansion coefficient to thermal conductivity. Materials like tungsten or molybdenum show desirable large ratios of $\lambda / \alpha$, and for the W7-X - CCRs molybdenum $\left(\lambda=138 \mathrm{~W} / \mathrm{m} \_\mathrm{K}\right.$, $\left.\alpha=5.7 \mathrm{e}-6 \mathrm{~K}^{-1}\right)$ is used. For instance, a heat flux of $\mathrm{q}=65 \mathrm{~kW} / \mathrm{m}^{2}$ through a plate of $\mathrm{d}=5 \mathrm{~mm}$ would lead to $\mathrm{R}=372 \mathrm{~m}$, which in this model is independent of the thickness of the plate. The corresponding temperature difference in this example is $\Delta \mathrm{T}=2.35 \mathrm{~K}$.

\subsection{Two dimensional heat transfer}

As seen from equation 1, the curvature of a heated plate of a given material is influenced by the ratio of temperature difference and plate thickness. In order to reduce the curvature it would be desirable to reduce the temperature difference for a given incident radiation and a fixed plate thickness. Fig. 2 shows a scheme for a finite difference calculation, which assumes a $2 \mathrm{D}$ heat transfer with lateral cooling. Plasma radiates to the top surface, the lower surface radiates to the plasma vessel, and at the left side the plate is kept at a constant temperature. The right boundary is adiabatic.

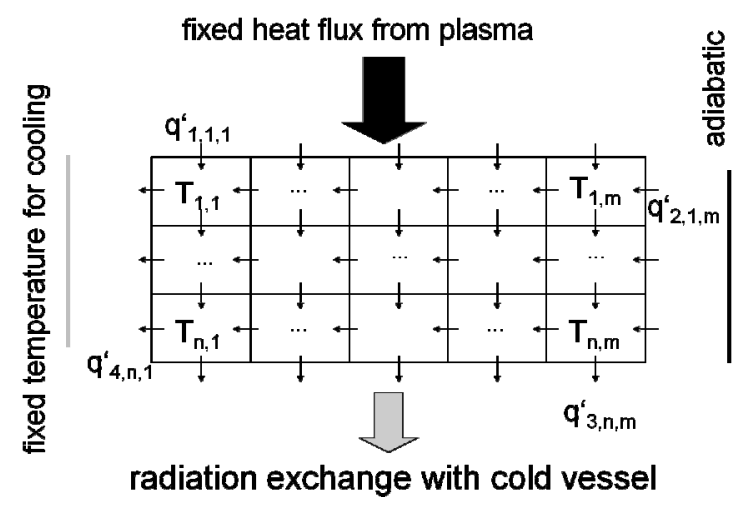

Fig. 2 Finite difference (FD) scheme for 2D heat transfer

For the same incident radiation as in 2.1 a constant temperature of $355^{\circ} \mathrm{C}$ is applied at the left boundary. This value is a result of a steady state calculation for the polarimetry CCR in ANSYS [5]. The temperature along the plate is then calculated with the presented FD scheme and the results are given in Fig. 3.The maximum difference between the temperatures of the top surface of the plate (solid line) and the lower surface (dotted line) is reduced to approximately $1 \mathrm{~K}$. According to equations (1) and (4) this leads to approximately twice the curvature radius which is advantageous for the CCR's function.

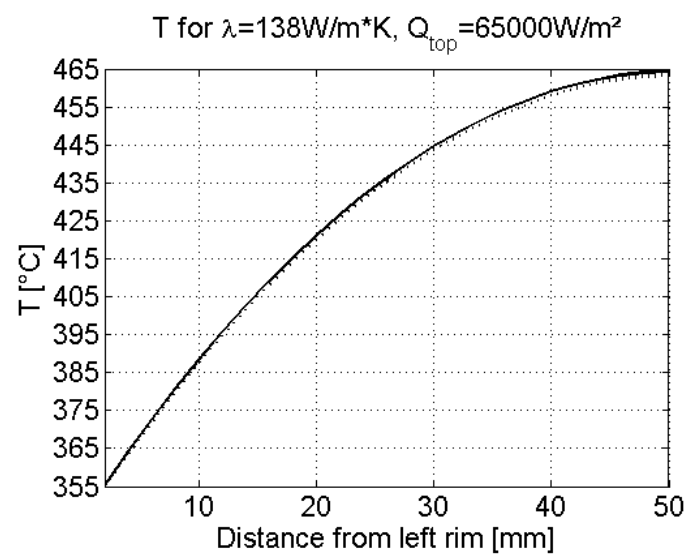

Fig. 3 Results of FD calculation for 2D heat transfer

Using the same setup it can also be shown that an increased thickness of the plate, albeit it reduces the overall temperature of the plate, does not affect the curvature in agreement with equation 3.

The cooling of all CCR plates in W7-X is similar to the $2 \mathrm{D}$ case described in this section. The cooling of the cube body (Fig. 4) is more complex, see below.

\section{Analyses}

ANSYS is used for coupled thermo-mechanical analyses of different CCRs used in the W7-X project. Fig. 4 shows the geometry of the polarimeter CCR, seen from the backside. The CCR is subjected to plasma radiation through an opening in the heat shields, and is cooled via its fixation at the heat sinks. This setup is 
similar for all CCRs in the project, even though the individual geometries differ according to the function of the CCR and the available space in the specific region.

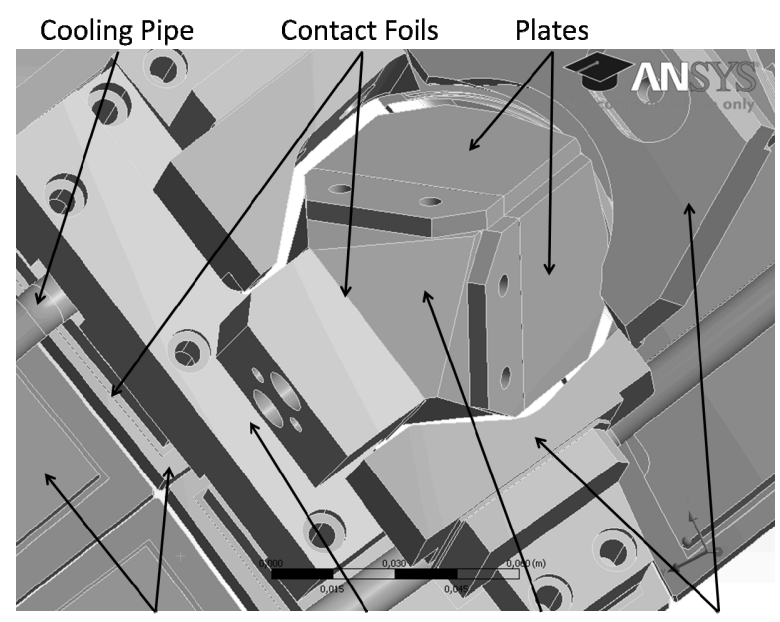

Heat Sink/Heat Shield Fixation Cube Body (CB) Shielding

Fig. 4 CCR for polarimetry seen from the plasma vessel

In a first step the temperature distribution is calculated taking into account thermal conduction via all contacts and thermal radiation between all parts. In a second step the resulting temperature distribution is used as initial thermal condition for a mechanical calculation which then yields total displacements for all parts. Finally, the local curvatures of the reflecting surfaces and changes in the angles between the surfaces are calculated with MATLAB [4].

\subsection{Polarimeter}

The thermal calculations for this CCR show a maximum temperature of $435^{\circ} \mathrm{C}$ at the top edges, which is acceptable. The regions of maximum heat fluxes in the fixation were identified (see Fig. 5), and this information was used to optimize its shape and consequently the cooling of the CCR.

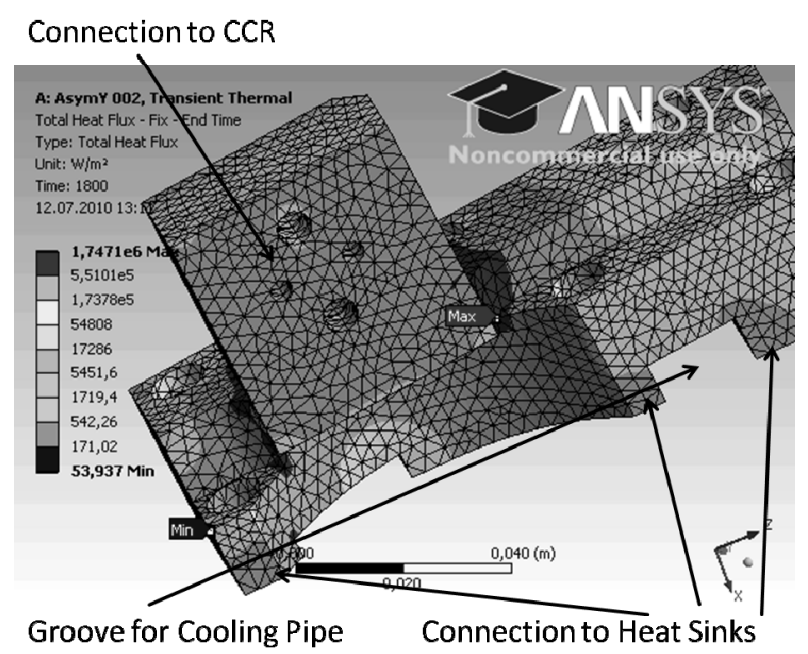

Fig. 5 Heat flux in CCR fixation

Using appropriate mechanical boundary conditions it is possible to visualize the local deformation of the reflecting surfaces in ANSYS (see Fig. 6).

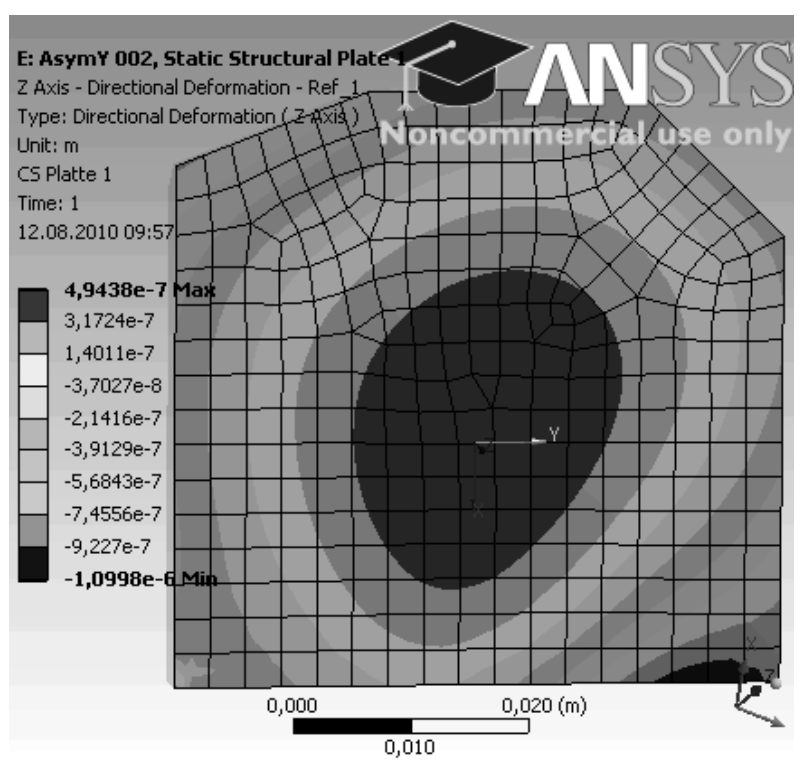

Fig. 6 Local deformation of one CCR plate

For a more detailed analysis, function fits along orthogonal axes through the maximum curvature of each reflecting surface were performed in MATLAB. The radius at the maximum curvature for each fitted function can then be calculated according equation 5 .

$$
r\left(x_{0}\right)=\left|\frac{\left(1+f^{\prime}\left(x_{0}\right)^{2}\right)^{\frac{3}{2}}}{f^{\prime \prime}\left(x_{0}\right)}\right|
$$

Since the surfaces are used to reflect Gaussian beams, also the average curvature radius within the beam cross section can be used. This can be approximated by equation 6 .

$$
r=\frac{h}{2}+\frac{s^{2}}{8 h}
$$

$\mathrm{r}$ - average radius of curvature

$\mathrm{h}$ - difference between locally lowest and highest point

$\mathrm{s}-$ diameter of beam

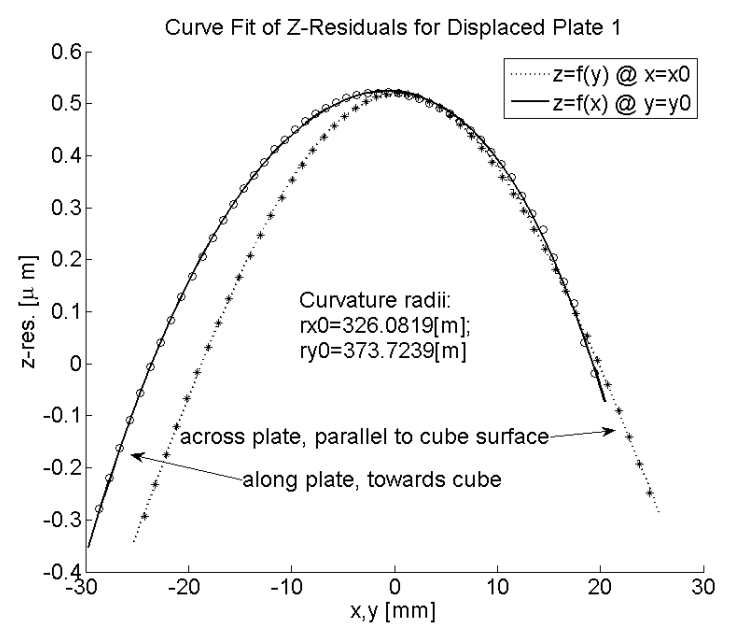

Fig. 7 Deformation and curvature radii of one CCR plate (x vertical, y horizontal in Fig. 6) 
The functions and curvature radii shown in Fig. 7 result from the deformation presented in Fig. 6.

Another MATLAB routine is used to create surface fits for all deformed reflecting surfaces and calculate their surface normals at different regions. Deviations from $90^{\circ}$ between normal vectors show how much the surfaces are tilted due to the thermal load which finally leads to a deviation from parallelism of the returning beam.

For the CCR used for polarimetry, the preliminary curvature radii for a plasma radiation of $80 \mathrm{~kW} / \mathrm{m}^{2}$ at an incident angle of $35.5^{\circ}$ are given in Table 1 . While the curvatures of the two plates are acceptable, the curvature of the cube body is still subject to further optimization (see section 4 ). The angles between all surfaces change by less than $0.02^{\circ}$.

Table 1: Curvature radii [m] of CCR surfaces for polarimetry

\begin{tabular}{lll}
\hline Part & $@ \mathrm{x}=\mathrm{x} 0$ & $@ \mathrm{y}=\mathrm{y} 0$ \\
\hline Plate 1 & 326 & 374 \\
Plate 2 & 362 & 236 \\
Cube Body & 151 & 60 \\
\hline
\end{tabular}

\subsection{Interferometer}

The same procedure as in 3.1 is also used for the ten interferometry CCRs. Due to the strong space restrictions at their locations and the smaller wavelength (106 $\mu \mathrm{m}$ from a CO2 laser) these CCRs will have a smaller aperture of about $25 \mathrm{~mm}$. The total heat flux, therefore, is less in spite of being positioned in a region of stronger plasma radiation. Even though the space for the fixations is extremely limited, the thermal connection to the heat sinks is good enough to keep the maximum temperature of the CCRs at approximately $230^{\circ} \mathrm{C}$. The curvature radii for an example with plasma radiation of $90 \mathrm{~kW} / \mathrm{m}^{2}$ at an incident angle of $35.5^{\circ}$ are given in Table 2. Again the angles between all surfaces change by less than $0.02^{\circ}$. Just like in case of the polarimetry CCRs, the curvatures of the cube body surfaces still need to be optimized (see 4).

Table 2: Curvature radii [m] of CCR plates for interferometry

\begin{tabular}{lll}
\hline Part & $@ \mathrm{x}=\mathrm{x} 0$ & $@ \mathrm{y}=\mathrm{y} 0$ \\
\hline Plate 1 & 834 & 253 \\
Plate 2 & 696 & 266 \\
Cube Body & 119 & 105 \\
\hline
\end{tabular}

\section{Optimization}

In all cases the reflecting surface of the cube body shows a stronger deformation than the two plates involved. This is due to the geometry and the distribution of heating and cooling. While the cube body receives additional heat at the sides where the plates are attached, it is well cooled at the centre, where it is connected to the fixation. This distribution occurs in addition to the plasma radiation which is more or less evenly distributed over the cube body's surface. The smallest radii occur at the centre of the surface where a concave curvature develops. However, it is possible to influence the distribution of heat fluxes in the cube body by shaping the form of the contact foils and thus the thermal connection between cube body and CCR fixation. As a proof of principle for one interferometry CCR, a hole in the centre of the contact foil between cube body and fixation was changed from $6.5 \mathrm{~mm}$ to $15 \mathrm{~mm}$ diameter in the ANSYS calculation. Just by directing the heat flux away from the centre of the cube body it was possible to decrease the curvature by approximately $30 \%$ (see Table 3 ). This approach will be used to optimize the shapes of the thermal contacts between the cube bodys and fixations.

Table 3: Curvature radii [m] of cube body surface depending on contact

\begin{tabular}{lll}
\hline Diameter of hole & $@, \mathrm{x}=\mathrm{x} 0$ & $@ \mathrm{y}=\mathrm{y} 0$ \\
\hline $6.5 \mathrm{~mm}$ & 235 & 174 \\
$15 \mathrm{~mm}$ & 337 & 224 \\
\hline
\end{tabular}

\section{Conclusions}

The W7-X project will use CCRs which each comprise a cube body and two reflector plates. A gap between the latter plates prevents mechanical contacts and thus the possibility of mutual deformations due to thermal expansion. Thermo-mechanical FE analyses show that the expected curvature radii of the reflector plates remain within acceptable limits for the given loads. The thermal conditions and the complex heat flux pattern in the cube bodys lead to smaller curvature radii of their reflecting surfaces. However, by shaping the thermal contact that connects the cube body to the cooling structure it is possible to direct heat fluxes and the corresponding temperature distribution in a way that results in smaller cube body surface deformations. This optimization still has to be performed for all CCRs.

$90^{\circ}$ angles between all three surfaces of a CCR are necessary to ensure the parallelism of the returning beam. It was shown that all angles change by less than $0.02^{\circ}$ which is acceptable.

\section{References}

[1] P. Kornejew, M. Hirsch, T. Bindermann, A. Dinklage, H. Dreier, H.-J. Hartfuß, Design of Multichannel Laser Interferometry for W7-X, Review of Scientific Instruments, 77 (2006), 10F128.

[2] M. Y. Ye, A. Werner, M. Hirsch, H. Thomsen, A. Weller, R. König, Thermal-Mechanical Analysis for in-Vessel Diagnostic Components in W7-X, AIP Conference Proceedings 993 (2008), 211-214

[3] M. Y. Ye, M. Hirsch, R. König, M. Laux, H. Thomsen, A. Weller, A. Werner, Thermo-Mechanical Analysis of Plasma Facing Components of Diagnostics in the Wendelstein 7-X Stellarator, Fusion Engineering and Design 84 (2009), 2002-2007

[4] MATLAB User's Manual, MathWorks Inc. MA, USA

[5] ANSYS User's Manual, ANSYS Inc. PA, USA 